\title{
Optic ataxia and the dorsal visual steam re-visited: impairment in bimanual haptic matching performed without vision
}

\author{
Stephen R. Jackson ${ }^{1}$, Laura A. Condon ${ }^{1}$, Roger W. Newport ${ }^{1}$, \\ Sally Pears ${ }^{1}$, Masud Husain ${ }^{2}$, Nin Bajaj ${ }^{1}$, Michael 0'Donoghue ${ }^{1}$ \\ University of Nottingham ${ }^{1}$ \\ and \\ University of Oxford ${ }^{2}$
}

Correspondence to:

Prof. Stephen Jackson

School of Psychology

The University of Nottingham

University Park

Nottingham

NG7 2RD, UK.

Stephen.jackson@nottingham.ac.uk

Key words:

Optic ataxia; Balint's syndrome; dorsal stream function; haptic matching; Simultanagnosia 


\section{Abstract}

The 'two visual systems' account proposed by Milner and Goodale (1992) argued that visual perception and the visual control of action depend upon functionally distinct and anatomically separable brain systems: a ventral stream of visual processing that mediates visual perception (object identification and recognition) and a dorsal stream of visual processing mediating visually guided action. Compelling evidence for this proposal was provided by the neuropsychological studies of brain injured patients, in particular the contrasting pattern of impaired and preserved visual processing abilities of the visual object agnostic patient (DF) and optic ataxic patients who it was argued presented with impaired dorsal stream function. Optic ataxia has thus become a cornerstone of this 'two visual system' account (Pisella, Sergio, Blangero, Torchin, Vighetto, Rossetti, 2009). In the current study we re-examine this assumption by investigating how several individuals presenting with optic ataxia performed on a bimanual haptic matching task performed without vision, when the bar to be matched was presented haptically or visually. We demonstrate that, unlike neurologically healthy controls who perform the task with high levels of accuracy, all of the optic ataxic patients were unable to perform the task. We interpret this finding as further evidence that the key difficulty experienced by optic ataxic patients across a range of behavioural tasks may be an inability to simultaneously and directly compare two spatial representations so as to compute the difference between them. 


\section{Introduction}

In their highly influential articles and books, Milner and Goodale proposed a distinction between a ventral stream of visual processing that mediated visual perception (object identification and recognition) and a dorsal stream of visual processing mediating visually guided action [e.g., Goodale \& Milner, 1992; Milner \& Goodale, 1992; Milner \& Goodale, 1995]. This 'two visual systems' account as it has come to be known argued that visual perception and the visual control of action depended upon functionally distinct and anatomically separable brain systems.

This 'two visual systems' proposal has proven to be immensely influential over the last two decades and, while this account was clearly based upon a considered review of a wide range of research findings from both humans and animals, it can be argued that its immediate and popular appeal stemmed from the compelling evidence drawn from neuropsychological studies of brain injured patients, in particular the contrasting pattern of impaired and preserved visual processing abilities of the visual object agnostic patient (DF) and optic ataxic patients. Thus, visual form agnosia has come to be viewed as the classic presentation associated with ventral stream damage whereas optic ataxia is viewed as the classic presentation following dorsal stream damage and a cornerstone of the two visual system account (Pisella, Sergio, Blangero, Torchin, Vighetto, Rossetti, 2009).

While the two visual systems account proposed by Milner and Goodale can be seen as a useful heuristic, several lines of evidence suggest that there may be cross-talk between the mechanisms responsible for visual perception and those responsible for visually guided action [e.g., Brenner \& Smeets, 1996; Jackson \& Shaw, 2000] and that optic ataxia should not be viewed as an impairment linked solely with dorsal stream damage [e.g., Himmelbach \& Karnath, 2005; Jackson, Newport, Mort, Husain, Jackson, Swainson, et al., 2005; Jackson, Newport, Mort, Husain, 2005; Jackson, Newport, Husain, Fowlie, O’Donoghue, \& Bajaj, 2009; Pisella, Sergio, Blangero, Torchin, Vighetto, Rossetti, 2009).

Optic ataxia [OA] was first described as a disorder of visually guided reaching movements that cannot be attributed to a basic motor or sensory deficit (Bálint, 
1909; Rizzo \& Vecera, 2002). The disorder was described initially by Bálint as one of a triad of visuospatial symptoms that can result from bilateral damage to the occipital-parietal cortex in humans (Bálint, 2009) and which has since become known as Bálint -Holmes or Bálint's syndrome (Rizzo \& Vecera, 2002). A key aspect of Bálint's view was that misreaching errors occurred as a consequence of disconnection between visual processing areas and motor regions responsible for planning reaching movements. More recent studies have confirmed that optic ataxia can follow unilateral damage to the parietal cortex of either hemisphere; most frequently involving the intraparietal sulcus and superior parietal lobule [SPL] or white matter underlying these areas (Perenin \& Vighetto, 1988).

In contrast to the view proposed by Bálint (1909), alternative contemporary accounts of optic ataxia argued that misreaching errors were not an independent, autonomous, symptom within Bálint's syndrome but instead arose as a consequence of impairments in visual perception (e.g., Holmes, 1918). Furthermore, recent studies have demonstrated that optic ataxic patients misreach to extra-foveal targets, irrespective of whether these targets are defined visually or in fact defined proprioceptively in the absence of vision (Blangero et al., 2007; Jackson et al., 2009).

Such observations, and in particular the key finding that optic ataxic patients typically only misreach when reaching to extra-foveal targets, led Jackson and colleagues to propose that reaching to an extra-foveal target may require additional processing steps that are not required when reaching to a foveated target, and that these additional processing steps may necessitate the simultaneous comparison of more than one visual and/or spatial representation (Jackson, Newport, Mort, Husain, Jackson, Swainson, et al., 2005; Jackson, Newport, Mort, Husain, 2005; Jackson et al., 2009). Specifically we argued that for extra-foveal reaching only, to compute a displacement vector in gaze-centred coordinates, it will first be necessary to simultaneously represent and compare the spatial location of the target in gaze-centered coordinates and the starting position of the hand in gaze-centered coordinates (Jackson et al., 2009). Importantly, this comparison may be necessary even where the target or hand location is specified initially in non-visual coordinates. This proposal was tested 
directly in a recent brain imaging study that hypothesized that the simultaneous representation of multiple spatial locations that must be directly compared with one another will involve increased metabolic costs relative to the case where only a single gaze-dependent location must be represented (Beurze, Toni, Pisella, \& Medendorp, 2010). The study confirmed this hypothesis by demonstrating that there were significant increases in brain activity within parietal and premotor areas of cortex for those movements that required the integration of peripheral target and hand positions within a gaze-centered frame. Based upon such evidence we have proposed that misreaching in optic ataxia may largely arise as a consequence of a limitation in the processing resources needed to simultaneously represent and compare more than one gaze-centred spatial representation (Jackson et al., 2009).

To further examine this issue we utilised a haptic matching task in the current study that we have reported previously (Newport, Rabb \& Jackson, 2002). Within this task participants are presented with a bar in a particular spatial orientation (the reference bar) and are then required to rotate a second bar (the test bar) to match the orientation of the first. To investigate the ability of optic ataxic patients to directly and simultaneously compare two spatial objects in the absence of vision, we conducted this haptic matching task in Experiment 1 as a bimanual matching task. In this case the participant, while wearing a blindfold, felt the orientation of the reference bar on each trial and was then required to rotate the test bar using their right hand to match the felt orientation of the reference bar. In Experiment 2 we varied the task by presenting the reference bar visually so that only one visual object was presented and could be foveated on each trial by the participant. In this case the participant's task was to rotate the unseen test bar using their right to match the orientation of the viewed visual reference bar. The results of these studies clearly demonstrated that whereas healthy controls could perform these matching tasks with a very high degree of accuracy, patients presenting with optic ataxia unable to perform that task.

\section{Experiment 1}

This experiment was adapted from the methods and procedures reported previously in Newport, Rabb \& Jackson (2002). 


\section{Methods}

\section{Control participants}

Eight naïve adult participants, aged between 19 and 34 years, participated in this study. All participants were right-handed and had normal or corrected-tonormal vision. No participant had any somatosensory impairment and all provided informed consent. An appropriate local ethical review committee approved the study.

\section{Patient II}

Patient was a 69 year old man who had been studied by our group over a lengthy period and had been the subject of several previous publications which had described in detail aspects of his Balint's syndrome [e.g., Jackson, Newport, Mort, \& Husain, 2005; Jackson, Shepherd, Mueller, Husain, \& Jackson, 2006; Newport, Brown, Husain, Mort, \& Jackson, 2006; Newport \& Jackson, 2006] A magnetic resonance imaging (MRI) scan had revealed asymmetrical bilateral atrophy to areas of the parietal, temporal and occipital cortices with damage to left parietal cortex extending further into the superior region. JJ experienced difficulty in seeing objects in his peripheral vision and frequently bumped into objects. JJ demonstrated a clear optic ataxia in that, when reaching for an object presented extra-foveally, he misreached by greater than $5 \mathrm{~cm}$. Furthermore, like Balint's original case report, JJ showed impairments largely with his right hand when pointing to extra-foveal targets presented in the left or right hemifield (Jackson et al. 2005). It should be noted that JJ's optic ataxia was tested regularly, over a 7-year period, and showed no change during that period.

JJ also exhibits clear evidence of attentional impairment, characterised by a profound simultanagnosia, when assessed using a number of tasks (Jackson, Shepherd, Mueller, Husain \& Jackson, 2006). For example, when presented with a complex visual display such as the 'Boston Cookie Theft' picture JJ can correctly report isolated items from the picture. For example, he will report seeing the following: "boy", "stool", "woman", "taps", "cup", "knife", "sink", "pot", but when he is asked to interpret what is happening in the picture, he fails to comprehend the picture globally and makes an incorrect guess of what is happening in the 
picture. Similarly, when presented with two visual stimuli, for example a pair of coloured line drawings or a pair of coloured letters, JJ will invariably report only one item from the each pair. Furthermore, on repeated testing with the same stimuli he will vary which of the two items that he reports, and will, on average, report each item equally often.

\section{Apparatus}

The apparatus consisted of two identical $8 \mathrm{~cm} \times 2 \mathrm{~cm}$ wooden bars, each mounted on a circular disc (diameter $10 \mathrm{~cm}$ ) that was itself mounted on a wooden board. One bar was located $15 \mathrm{~cm}$ to the left of the participant's midsagittal axis and the other $15 \mathrm{~cm}$ to the participant's right. On each trial one bar would serve as the 'reference bar' while the other served as the 'test bar'. For control participants the right hand bar served as the reference bar and the left hand bar the test bar. For patient JJ, the right and left hand bars each served as the reference bar (in separate sessions) and the other bar served as the test bar. Throughout the experiment each participant was seated directly in front of the wooden board that was situated at a comfortable reaching distance for that participant. In Experiment 1 participants were blindfolded throughout and their hands were placed upon the test and reference bars at the start of each trial by the experimenter.

\section{Design and Procedure}

On each trial the 'reference' bar could be presented, in a pseudorandom order, at one of four orientations relative to the participant's mid-sagittal axis $\left(0^{\circ}\right)$. These orientations were $-60^{\circ},-20^{\circ},+20^{\circ}$, and $+60^{\circ}$ (see Figure $1 \mathrm{~A}$ ). The other bar, the 'test' bar, was then presented at an orientation of $0^{\circ}$ at the beginning of each trial (see Figure 1A). The participant's task on each trial was to rotate the test bar (using the hand ipsilateral to the test bar) so that it was either parallel (Figure 1B) or mirror-symmetrical (Figure 1C) to the orientation of the reference bar. The angles of the reference and test bars were recorded with a Minibird electro- magnetic motion-tracking device and analysed off-line using in-house software programmed using Matlab. 


\section{Figure 1 about here}

\section{Results}

To investigate participants' accuracy in matching (parallel match or mirror symmetrical match) the reference angle linear regression and Pearson correlation analyses were calculated for each participant and for each type of match (i.e., parallel or mirror symmetrical). These analyses yielded slope and goodness-of-fit ( $\mathrm{R}^{2}$ - variance explained) values, Pearson correlation coefficient values, and associated t-scores and p-values. These data are presented in Table 1 and representative graphical data is presented in Figure 2.

\section{Table 1 and Figure 2 about here}

These data clearly confirm previous reports (e.g., Newport, Rabb, Jackson, 2002) that neurologically healthy control participants can perform this haptic matching task with extremely high levels of consistency and accuracy. In the current study the angle set by healthy controls on each trial was highly correlated with the reference angle set on that trial (Means: parallel match $=$ 0.98; mirror symmetrical match $=0.98$; range $=0.97-0.99$ ) and the reference angle accounted for almost all of the variance in the set angle (Mean $\mathrm{R}^{2}$ values: parallel match $=0.96$; mirror symmetrical match $=0.96$; range $=0.93-0.97$ ). In all cases these effects were statistically significant $(\mathrm{p}<0.001)$. By contrast, the findings from patient JJ indicate that he was unable to perform the haptic match task, either for the parallel or mirror symmetrical match, with any degree of accuracy (see Figure 2 and Table 1). Specifically, the set angles produced by JJ on each trial were uncorrelated with the reference angles (parallel match $=0.04, \mathrm{R}^{2}$ $<0.1$, t-value $=0.3, \mathrm{p}>0.1$; mirror symmetrical match $=-0.05, \mathrm{R}^{2}<0.1$, $\mathrm{t}$-value $=$ $0.4, \mathrm{p}>0.1)$.

\section{Discussion}

Previous studies, using a bimanual haptic matching task, have demonstrated that neurologically healthy adults can accurately match the felt orientation of a target object in the absence of vision (e.g., Newport, Rabb \& Jackson, 2002). In 
the current study we investigated whether Patient JJ, who presents with Balint's syndrome following repeated strokes, can accurately perform the bimanual haptic matching task in the absence of vision. As previously reported, JJ presents with a persistent and well-described optic ataxia which is characterised as a misreaching to visually defined targets, particularly when these are presented extra-foveally (i.e., away from the point of current fixation), and when reaching movements are executed using his right hand.

As the classic description of optic ataxia is misreaching to visually defined targets, but with preserved reaching accuracy for proprioceptively defined targets (Bálint, 1909; Buxbaum \& Coslett, 1997; Rizzo \& Vecera, 2002), we reasoned that JJ's performance on the bimanual haptic matching task ought to be relatively normal when movements were executed in the absence of vision. However this was clearly not the case as JJ exhibited a severe impairment on the task, irrespective of whether he was required to carry out a parallel or a mirror symmetrical match.

As noted above, alternative accounts of optic ataxia have argued that misreaching errors are not an independent, autonomous, symptom but may instead arise as a consequence of impairments in visual perception (e.g., Holmes, 1918), and recent studies have demonstrated that optic ataxic patients misreach to extra-foveal targets, irrespective of whether these targets are defined visually or in fact proprioceptively (Blangero et al., 2007; Jackson et al., 2009) leading us to propose that misreaching in optic ataxia may largely arise as a consequence of a limitation in the processing resources needed to simultaneously represent and compare more than one gaze-centred spatial representation (Jackson et al., 2009). We interpret the current finding, that patient JJ could not match the orientation of two haptically defined objects when these are presented entirely without vision, as broadly consistent with the above proposal.

\section{Experiment 2}

To further investigate this issue we conducted a revised version of the haptic matching experiment in which the orientation of the reference bar could be presented either haptically (as described in Experiment 1) or visually (i.e., the 
participant saw a single visual bar and then, out of sight, haptically adjusted the test bar to match the orientation of the [visual] reference bar).

Our reasoning for this manipulation was as follows: if, as argued by Holmes (1918), optic ataxia is not an independent symptom but instead arises due to perceptual impairment characterised as an inability to relate visual objects to one another and combine visual components into a coherent whole (i.e. simultanagnosia), then presenting optic ataxic patients with only a single visual object in view, which they can foveate throughout, ought to resolve their inability to complete the haptic matching task. This is consistent with the oft-reported finding that optic ataxic patients can reach accurately to foveated objects but misreach to targets presented away from the point of fixation (e.g. Buxbaum \& Coslett, 1997; Jackson et al., 2005).

In addition to this change in task, we also sought to replicate the effects observed for patient JJ in Experiment 1 by testing additional patients, with mixed aetiologies, who also presented with Balint's syndrome, including a clearly observed optic ataxia.

Finally, to accommodate changes in the design and procedure, a new method of measuring and recording the orientation of the reference and test bars was adopted in Experiment 2 (i.e., the use of the Minibird electromagnetic recording system was replaced with the use of potentiometers to record the orientation of the reference and test bars).

\section{Methods}

\section{Control participants}

Twelve neurological healthy adults participated in the study. All were righthanded and either undergraduate or postgraduate students ( 6 male, 6 female) at the University of Nottingham (mean age $=23$ ). All were right-handed, naïve to the design and purpose of the study, and had normal or corrected to normal vision.

\section{Balint's syndrome patients}

Six patients were recruited to this study ( 1 female [MN]). The patients ranged in age from 61 - 77 years. All of the patients had been referred to a neurologist 
with visuospatial problems and had been thoroughly investigated. Five of the patients (Cases 1-5) presented with Balint's syndrome, characterised by a clear an unambiguous optic ataxia (affecting one or both upper limbs) and severe simultanagnosia (assessed using a variety of neuropsychological tests including the BORB (Humphreys \& Riddoch, 1993). Three of these cases (Case 2 [JY], Case 3 [MN], and Case 5 [JJ]) have been reported previously. The cases had mixed aetiologies. Cases 1-4 had been given a clinical diagnosis posterior cortical atrophy [PCA]. Figure 3 illustrates MRI scans for cases 2 [JY] and 3 [MN]. Case 5 [JJ], as noted above, had suffered a number of haemorrhagic strokes over a period of several years leading to asymmetrical bilateral atrophy to parietal, temporal and occipital cortices with damage to left parietal cortex extending further into the superior region.

Figure 3 about here

In addition to testing for perceptual and visuomotor impairment, all of the above cases were tested specifically for somatosensory dysfunction and intellectual impairment. In all cases, formal assessment of upper-limb somatosensory and proprioceptive function revealed no somatosensory or proprioceptive impairment. Similarly, general intellectual abilities (e.g., language, verbal IQ, understanding of verbal problems, and executive function) were also unimpaired in all cases. Finally, in all cases it was established prior to testing that readily see and identify the single visual bar when it was presented on the computer monitor.

Control patient $[\mathrm{HH}]$

HH was a 69-year-old right-handed male who was referred to a neurologist with visuospatial problems following a stroke. Neurological examination and neuropsychological assessment of $\mathrm{HH}$ revealed that his intellectual function was normal but he presented with a mild left hemispatial neglect. Specific testing of his somatosensory and motor function revealed that his somatosensory, proprioceptive, and motor functions were normal for both upper limbs, and specifically that he showed no evidence of optic ataxia for either limb. Perceptual testing, including use of the BORB (ref), revealed no evidence of simultanagnosia. 
Based upon these assessments, HH was recruited as a patient control, particularly for Case 5 [JJ] in view of their similar aetiology.

\section{Apparatus}

The apparatus consisted of a pair of plastic bars, each mounted on a plastic disc that could rotate within a plastic block. Each disc contained a potentiometer that recorded a voltage that corresponded to the orientation of the bar. The centres of the two discs were placed $26 \mathrm{~cm}$ apart upon a black veneered table. All participants were required to sit at the table so that the potentiometers were situated equidistantly to the left and right of their mid-sagittal axis. For proprioceptive haptic matching trials the bar situated to the left of the midline was the reference bar, and the bar situated to the right served as the test bar.

A moveable wooden box containing a flat LCD screen was placed above the potentiometers. The box was painted black so as to remain congruent with the workspace and embedded within the box was a flat LCD screen situated on the left hand side of the box immediately above the location of the reference bar (see Figure 4). The box was elevated $15 \mathrm{~cm}$ above the workspace allowing the screen to appear at an appropriate distance for the participant to both feel and move the potentiometers comfortably underneath, and to allow a comfortable viewing distance from the participant's midline.

\section{Figure 4 about here}

Both bars could be comfortably rotated between the angles of $30^{\circ}$ and $150^{\circ}$, relative to the participant's sagittal axis $\left(90^{\circ}\right)$. Both potentiometers were calibrated prior to each recording session and voltages from each potentiometer continuously recorded throughout each trial using in-house software written in Matlab. Data were then analysed offline using in-house software written in Matlab.

\section{Procedure}

The experiment was a repeated-measures design that comprised of two conditions that were executed in separate sessions: a vision-to-proprioception condition (VP) and a bimanual proprioception-to-proprioception (PP) haptic match condition (as described in Experiment 1 above). 
In both conditions, at the start of each trial, the test bar was placed at an orientation of $90^{\circ}$ by the experimenter and participants were then required to rotate the test bar so that it was either parallel to, or, mirror symmetrical to, the angle of the reference bar. Participants were given a period of five seconds to complete this movement.

In the VP condition participants rotated the test bar angle from its $90^{\circ}$ starting position to match the perceived angle of a visual reference bar displayed on the LCD screen. The presentation of the reference angle was generated randomly by an in-house Matlab programme. Throughout the VP sessions the participant's vision of the test bar and their right upper limb was obscured by a black cloth.

In the PP condition the virtual screen was removed and participants had to match the test bar angle to the angle of the reference bar that was perturbed pseudorandomly by the experimenter. The participant's hand was placed on the reference bar at the start of each trial and then removed so that the experimenter could move the bar to a new orientation. To ensure that the participants were acting upon proprioceptive information alone they were blindfolded throughout. In both the VP and PP conditions participants were not given feedback on their performance.

All of the control participants performed each condition (VP and PP) twice: once to make the test bar parallel to the reference bar/virtual reference bar angle, and once to make the test bar angle mirror symmetrical to the reference bar/virtual reference bar angle. The order of these sessions was randomised across participants. Each condition consisted of 5 practice trials followed by 30 experimental trials. Each participant completed a total of 120 experimental trials. As this procedure was rather lengthy we found that the patients could not complete the whole experiment in one visit. For this reason patients completed either the parallel match (VP and PP conditions) or the mirror symmetrical match session during their visit (a total of 60 trials).

\section{Tables 2 and 3 about here}

\section{Results}


To investigate participants' accuracy in matching (parallel match or mirror symmetrical match) the visually or haptically presented reference angle, separate linear regression and Pearson correlation analyses were calculated for each participant and for each type of match (i.e., parallel or mirror symmetrical). These analyses yielded slope, goodness-of-fit ( $\mathrm{R}^{2}$ - variance explained), and 95\% confidence interval values, Pearson correlation coefficient values and associated p-values.

\section{Figure 5 about here}

Data from the group of healthy control participants are presented in Tables 2 and 3 and representative graphical data from a single individual is presented in Figure 5. Inspection of these data clearly demonstrate that the control participants are very accurate at rotating the test bar to match the reference bar, irrespective of whether the reference bar orientation was presented visually or haptically or whether the task was to make the test bar parallel or mirror symmetrical to the reference bar (group mean correlations: haptic parallel match $=0.99$; visual parallel match $=0.98$; haptic mirror symmetrical match $=0.98$; visual mirror symmetrical match $=0.98$; all $\mathrm{p}<0.0001$ ).

\section{Table 4 about here}

Data from the patients are presented in Table 4. Inspection of this table reveals that the age-matched control patient $\mathrm{HH}$, who does not present with optic ataxia or simultanagnosia but instead a mild hemispatial neglect, can perform the haptic matching task extremely well and at a level comparable to the young health controls bar (mean correlations: haptic parallel match $=0.99$; visual parallel match $=0.95$; both $\mathrm{p}<0.001$ ). By contrast, all five of the patients presenting with optic ataxia Balint's syndrome and were unable to perform the haptic matching task irrespective of whether the reference bar orientation was presented haptically without vision (PP) or when a single visual reference bar was presented (VP). Representative graphical data from three individual patients are presented in Figure 6.

\section{Discussion}


We reasoned that if misreaching errors in optic ataxia were not an independent symptom, as argued by Holmes (1918), but instead arose due to perceptual impairment characterised to an inability to relate visual objects to one another and combine visual components into a coherent whole (i.e. simultanagnosia), then presenting optic ataxic patients with only a single visual object to foveate ought to resolve their inability to complete the haptic matching task. However, this was not the case for optic ataxic patients tested in the current study who were unable to complete the haptic matching task irrespective of whether the reference bar was presented haptically without vision or as a single visual object that they could foveate throughout. By contrast, an age-matched patient presenting with mild hemispatial neglect could complete both tasks as well as a group of healthy control participants.

Based upon these findings we take the view that the difficulty experienced by the optic ataxic patients due to the requirement in both tasks to simultaneously and directly compare two spatial orientations so as to compute the difference between them. The current data suggest that visual presentation of the stimuli is not a necessary requirement for this impairment to occur and that presenting a single visual stimulus to foveate is not sufficient to produce accurate performance.

\section{General Discussion}

As noted above, optic ataxic patients typically exhibit misreaching errors only when reaching to extra-foveal target locations (Buxbaum and Coslett, 1997) and recent studies have demonstrated that optic ataxic patients misreach when reaching for extra-foveal targets, irrespective of whether these targets are defined visually or in fact proprioceptively (Blangero et al., 2007; Jackson et al., 2009). We proposed that misreaching in optic ataxia might arise as a consequence of a limitation in the processing resources needed to simultaneously represent and directly compare more than one gaze-centred spatial representation (Jackson et al., 2009).

In the current study we tested this general idea by investigating how optic ataxic patients performed on a non-reaching task, specifically a haptic matching task that required them to match the orientation of an unseen object (the test 
bar) with that of a second object that was either viewed directly (Experiment 2) or else unseen but felt using the other hand (Experiments 1 and 2). In both experiments we clearly demonstrated that neurologically healthy individuals could consistently perform this task with a high degree of accuracy thus confirming previous demonstrations using this task (e.g., Newport, Rabb \& Jackson, 2002). By contrast, patients presenting with optic ataxia were unable to perform this task producing responses (i.e., set bar orientations) that were uncorrelated with the reference bar orientations presented on each trial. Furthermore, this was the case irrespective of whether the task was performed without vision (i.e., the participant was blindfold throughout and matched the orientations of the test and reference bars based upon haptic and proprioceptive information alone) or a single visual object (reference bar) was displayed. These findings are discussed below.

The classic description of Optic ataxia, as initially reported by Bálint (1909) and then subsequently described by many others (Rizzo \& Vecera, 2002), is of an impairment of reaching to visually defined targets, which Bálint viewed as arising from the disconnection between visual processing areas and motor regions responsible for planning reaching movements. Key to this description was the demonstration that optic ataxic patients could execute accurate reaching movements to non-visual, proprioceptively defined, target locations (e.g., they could reach accurately without vision to touch their own body parts). This suggested to us that, in the absence of vision, optic ataxic patients ought to be unimpaired at performing the bimanual haptic matching task, particularly the mirror symmetrical matching condition, as this explicitly requires the participant to match postural information across the arms (Newport, Rabb \& Jackson, 2002). However, this was not the case as the patients we tested we equally poor at both parallel matching and mirror symmetrical matching.

An alternative contemporary account of optic ataxia provided by Holmes (1918) was to reject the notion of misreaching as a separate and independent symptom in favour of the idea that the misreaching in fact arose as a consequence of a perceptual impairment characterised by an inability to relate visual objects to one another and combine visual components into a coherent 
whole (i.e. what we would now refer to as simultanagnosia). All of the optic ataxic patients reported in the current study presented with Bálint's syndrome and thus exhibited both simultanagnosia and optic ataxia. Simultanagnosia is a disorder of visual perception characterised by the inability to interpret complex visual arrays despite preserved recognition of single objects (Coslett \& Saffran, 1991). It is often referred to as an inability to see more than one object at a time (Robertson, 2003) and is thought to occur as a consequence of dysfunctional mechanisms of visual attention (e.g., Robertson, 2003) or visual awareness (Jackson, Shepherd, Mueller, Husain \& Jackson, 2006).

Given the above description of simultanagnosia, it is unclear why our patients, even if they do present with simultanagnosia, should perform the haptic matching tasks so very poorly. In the current study our optic ataxic patients were required to perform either a bimanual haptic matching task while wearing a blindfold throughout the study (Experiments $1 \& 2$ ), and thus relied throughout on haptic and proprioceptive information to perform the task, or, in an amended version of the haptic matching task (Experiment 2), the orientation of the reference bar was presented visually and the participant matched this orientation by rotating the unseen test bar with their right hand. In this case only one visual object was ever presented, and all of our patients were unimpaired at identifying single visual objects. We note that previous studies have identified a line orientation deficit (e.g., Mehta \& Newcombe, 1991) in which patients could not determine if two visually presented lines matched. This was not explicitly tested in the current study but we acknowledge that it might form an additional component of the deficit we observed.

We have suggested previously (e.g., Newport \& Jackson, 2006; Jackson et al., 2009) that the key difficulty experienced by optic ataxic patients is an inability to simultaneously and directly compare two spatial representations so as to compute the difference between them (e.g., comparing the eye-centred location of target and hand during non-foveal reaching so as to compute an appropriate displacement vector, or comparing the unseen orientation of the reference and test bars in the current study). While this appears highly reminiscent of simultanagnosia, it could not currently be included as an instance of 
simultanagnosia unless the definition of that disorder were broadened to include an inability to simultaneously represent, within the 'mind's eye', more than one spatial representation, and thereby extended to include the representation of objects that were initially presented non-visually.

One important caveat with respect to our findings is that all of the optic ataxic patients included in our study presented with Bálint's syndrome following bilateral damage to occipital -parietal regions of the cortex. Such cases are similar to the patient originally reported by Bálint and are far more common that cases in which optic ataxia occurs following unilateral brain damage (Perenin \& Vighetto, 1988). It has been suggested that optic ataxic patients with unilateral and bilateral damage may differ, and it has been proposed that optic ataxic patients with bilateral damage may also present with simultanagnosia leading to reaching difficulties for foveated objects (Pisella et al., 2009). It should be noted that all of the patients reported in the current study, despite their simultagnosia, had absolutely no difficulty in accurately reaching for foveated objects, and only exhibited reaching errors when reaching for extra-foveal targets. It remains to be seen whether optic ataxic patients with unilateral lesions can perform the haptic matching tasks described in our paper.

One longstanding argument against the view that perceptual impairment contributes to the misreaching in optic ataxia comes from Bálint's original description of a 'hand effect' in his patient, i.e., misreaching for a visual target with one limb but not the other. However, the existence of the so-called hand effect does not adequately explain accurate reaching movements made using the 'impaired hand' for foveated objects, but highly erroneous reaching to extrafoveal objects (the most common presentation of optic ataxia), or why reaching errors are almost always in the direction of the point of current fixation (see Jackson et al., 2005).

In summary, the 'two visual systems' account comprising of a ventral stream of visual processing that mediated visual perception (object identification and recognition) and a dorsal stream of visual processing mediating visually guided action (e.g., Goodale \& Milner, 1992; Milner \& Goodale, 1992; Milner \& Goodale, 1995) has proven to be an extremely useful heuristic for thinking visual 
processing in the context of action. However, several lines of evidence suggest that there may be cross talk between the mechanisms responsible for visual perception and those responsible for visually guided action (e.g., Brenner \& Smeets, 1996; Jackson \& Shaw, 2000), and recent articles has suggested that this proposal may be an oversimplification (e.g., Pisella et al., 2009). While, optic ataxia has been viewed as the classic neuropsychological impairment following dorsal stream damage, and as such is seen as the cornerstone of the two visual system account (Pisella et al., 2009), the findings of this study suggest that this is very likely an unhelpful oversimplification of the case, at least for the vast majority of patients who present with optic ataxia as part of Bálint's syndrome.

\section{Acknowledgements}

We are extremely grateful to all of the patients who participated in this study and to their families. We thank Jane Fowlie for her help with patient recruitment and administration. We thank Nancy Weir for her help in developing the figure of the apparatus used in Experiment 2.

\section{References}

Bálint, R. (1909). Seelenlähmung des 'schauens', optische ataxie, räumliche störung der aufmerksamkeit. Monattsschrifte für Psychiatrische Neurologie 25: 51-81.

Beurze SM, Toni I, Pisella L, \& Medendorp WP. (2010). Reference Frames for Reach Planning in Human Parietofrontal Cortex. Journal of Neurophysiology 104: 1736-1745.

Blangero, A., Ota, H., Delporte, L., Revol, P., Vindras, P., Rode, G., et al. (2007). Optic ataxia is not only 'optic': Impaired spatial integration of proprioceptive information. NeuroImage, 36, T61-T68.

Brenner, E., \& Smeets, J, B. J. (1996). Size illusion influences how we lift but not how we grasp an object. Experimental Brain Research, 111, 473-476.

Buxbaum, L. J., \& Coslett, H. B. (1997). Subtypes of optic ataxia: Reframing the disconnection account. Neurocase, 3, 159-166. 
Coslett HB, Saffran E. (1991). Simultanagnosia: To see but not two see. Brain 114, 1523-45.

Goodale, M. A., \& Milner, A. D. (1992). Separate visual pathways for perception and action. Trends Neuroscience, 15(1), 20-25.

Himmelbach, M., \& Karnath, H. O. (2005). Dorsal and ventral stream interaction: Contributions from optic ataxia. Journal of Cognitive Neuroscience, 17, 632640.

Holmes, G. (1918). Disturbances of visual orientation. British Journal of Ophthalmology 2: 449-68, 506-16.

Humphreys G \& Riddoch MJ., Birmingham Object Recognition Battery, 1993. HoveLawrence Erlbaum Associates

Jackson, S. R., Newport, R., Husain, M., Fowlie, J. E., O’Donoghue, M., \& Bajaj, N. (2009). There may be more to reaching than meets the eye: Re-thinking optic ataxia. Neuropsychologia, 47(6), 1397-1408.

Jackson, S. R., Newport, R., Mort, D., Husain, M., Jackson, G. M., Swainson, R., et al. (2005). Action binding and parietal lobes: some new perspectives on optic ataxia. In G. W. Humphreys, \& M. J. Riddoch (Eds.), Attention in action: Advances from cognitive neuroscience. Hove UK: Psychology Press.

Jackson SR, Newport R, Mort D, Husain M. (2005). Where the Eye Looks, the Hand Follows: Limb-Dependent Magnetic Misreaching in Optic Ataxia. Current Biology 15, 42-46.

Jackson SR \& Shaw A (2000). The Ponzo Illusion Affects Grip-Force But Not GripAperture Scaling During Prehension Movements. Journal of Experimental Psychology: Human Perception and Performance 26(1): 418-423.

Jackson GM, Shepherd T, Mueller SC, Husain M, and Jackson SR (2006). Dorsal Simultanagnosia: An impairment of visual processing or visual awareness? Cortex 5, 740-749.

Mehta Z \& Newcombe F. (1991). A role for the left hemisphere in spatial processing. Cortex 27: 153-167.

Milner, A. D., \& Goodale, M. A. (1992). Trends in Neurosciences, 15, 20-25.

Milner, A. D., \& Goodale, M. A. (1995). The visual brain in action. Oxford: Oxford University Press.

Newport R, Brown L, Husain M, Mort D, Jackson SR. (2006). The role of the 
posterior parietal lobe in prism adaptation: failure to adapt to optical prisms in a patient with bilateral damage to posterior parietal cortex. Cortex 5,720729.

Newport R and Jackson SR. (2006). The posterior parietal cortex and prism adaptation. Neuropsychologia 44: 2757-2765.

Newport R, Rabb B, Jackson SR. (2002). Non-informative vision improves haptic spatial perception. Current Biology 12: 1661-1664.

Perenin, M. T., \& Vighetto, A. (1988). Optic ataxia: a specific disruption in visuomotor mechanisms. I. Different aspects of the deficit in reaching for objects. Brain, 111, 643-674.

Pisella L, Sergio L, Blangero A, Torchin H, Vighetto A, Rossetti Y. (2009). Optic ataxia and the function of the dorsal stream: Contributions to perception and action. Neuropsychologia 47: 3033-3044.

Rizzo, M., \& Vecera, S. P. (2002). Psychoanatomical substrates of Bálint's syndrome. Journal of Neurology, Neurosurgery and Psychiatry, 72, 162-178.

Robertson LC. (2003). Binding, spatial attention and perceptual awareness. Nature Neuroscience 4: 93-102. 
Figure Captions:

Figure 1: Graphical representation of the haptic matching task. A. illustrates the locations of the test and reference bars and that the reference bar could be set at different orientations on each trial. B. illustrates how the two bars might be aligned in the parallel match condition. C. illustrates how the two bars might be aligned in the mirror symmetrical match condition.

Figure 2: Representative data illustrating the accuracy of the relationship between the reference angle and the angle set by the participant. A. illustrates data from four representative healthy controls in the parallel match condition. B. illustrates data from the same four representative healthy controls in the mirror symmetrical match condition. C/D. Patient JJ's performance on the bimanual haptic match task using his left (C.) and right (D.) hand in the parallel match condition. E/F. Patient JJ's performance on the bimanual haptic match task using his left (E.) and right (F.) hand in the mirror symmetrical match condition.

Figure 3: The upper panel shows a coronal and axial view of patient JY's MRI scan which illustrates substantial atrophy particularly involving the left parietal cortex. The lower panel shows axial and coronal views of patient MN's MRI scan which also illustrates substantial atrophy particularly involving the left parietal cortex.

Figure 4: Graphical representation of the apparatus used in Experiment 2 (see text for further details).

Figure 5: Illustrates representative data from a single healthy control participant. a. Association between the visual reference bar angle and the haptic set bar angle in the parallel match condition. b. Association between the haptic reference bar angle and the haptic set bar angle in the parallel match condition. c. Association between the visual reference bar angle and the haptic set bar angle in the mirror symmetrical match condition. d. Association between the haptic reference bar angle and the haptic set bar angle in the mirror symmetrical match condition.

Figure 6: Illustrates representative data from three representative optic ataxic patients. Upper panel: Case 1 [GO]; a. Association between the visual reference bar angle and the haptic set bar angle in the mirror symmetrical match condition. b. Association between the haptic reference bar angle and the haptic set bar angle in the mirror symmetrical match condition. Middle panel: Case 2 [JY]; a. Association between the visual reference bar angle and the haptic set bar angle in the mirror symmetrical match condition. $\mathbf{b}$. Association between the haptic reference bar angle and the haptic set bar angle in the mirror symmetrical match condition. Lower panel: Case 4 [RP]; a. Association between the visual reference bar angle and the haptic set bar angle in the parallel match condition. b. Association between the haptic reference bar angle and the haptic set bar angle in the parallel match condition. 
Figure 1

A.

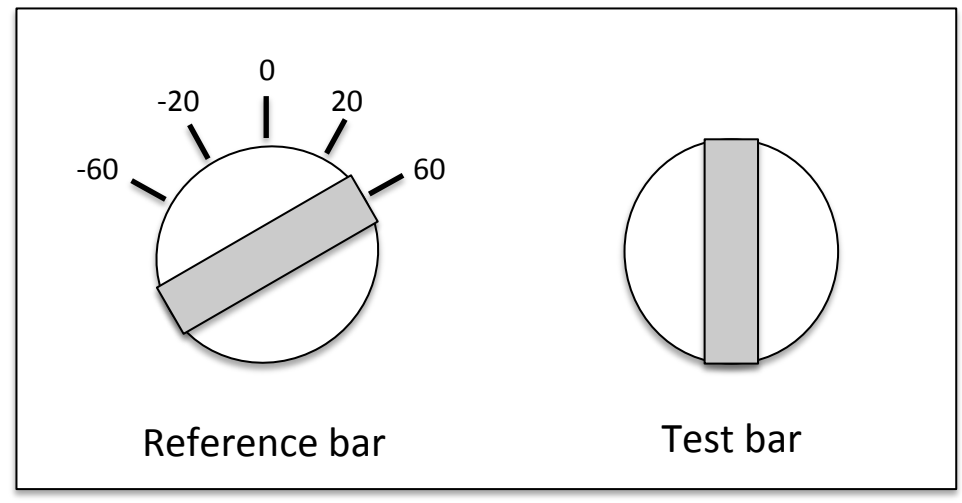

B.

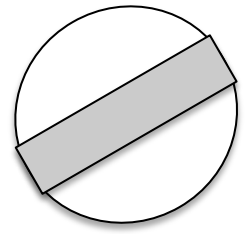

Test bar

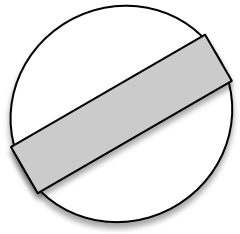

Reference bar

C.

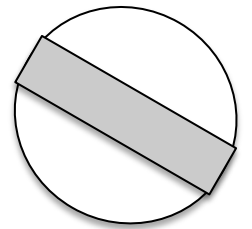

Test bar

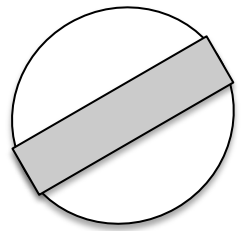

Reference bar 
Figure 2

A.
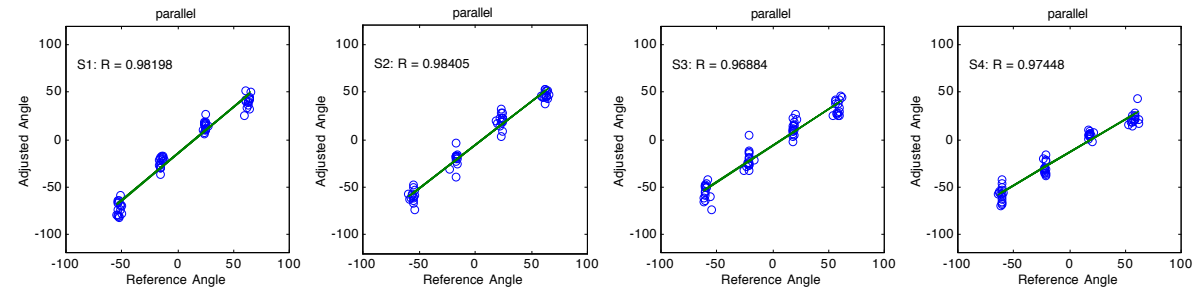

B.
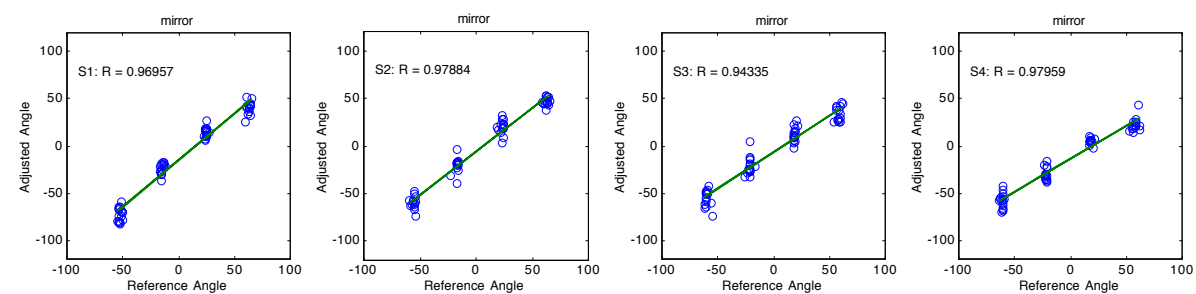

C.

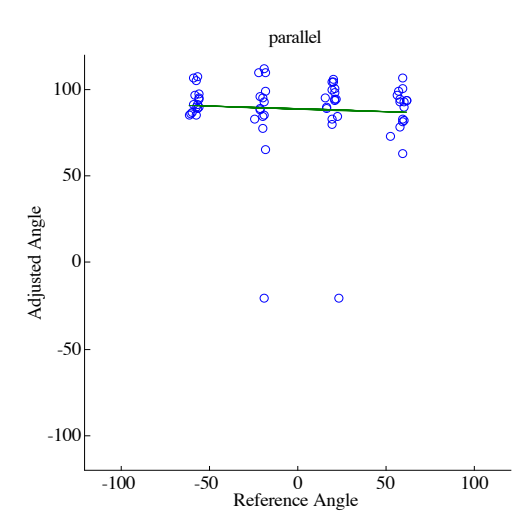

D.

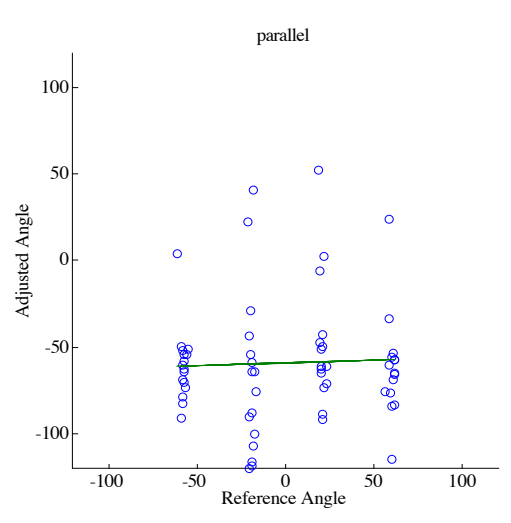

E.

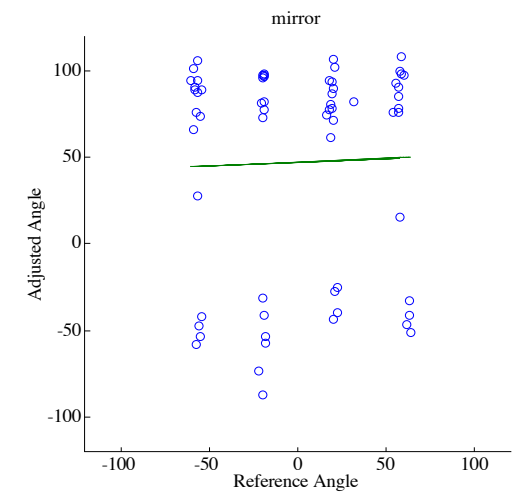

F.

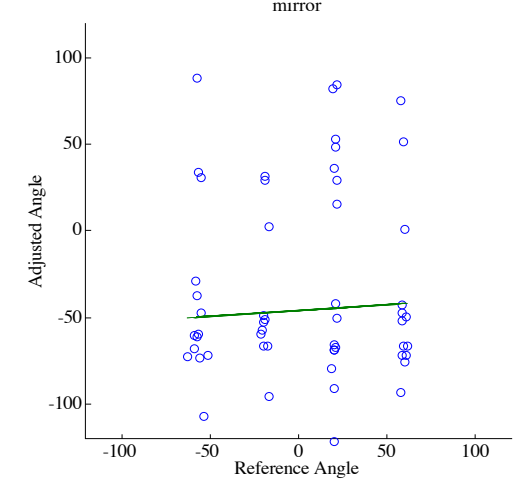


Figure 3
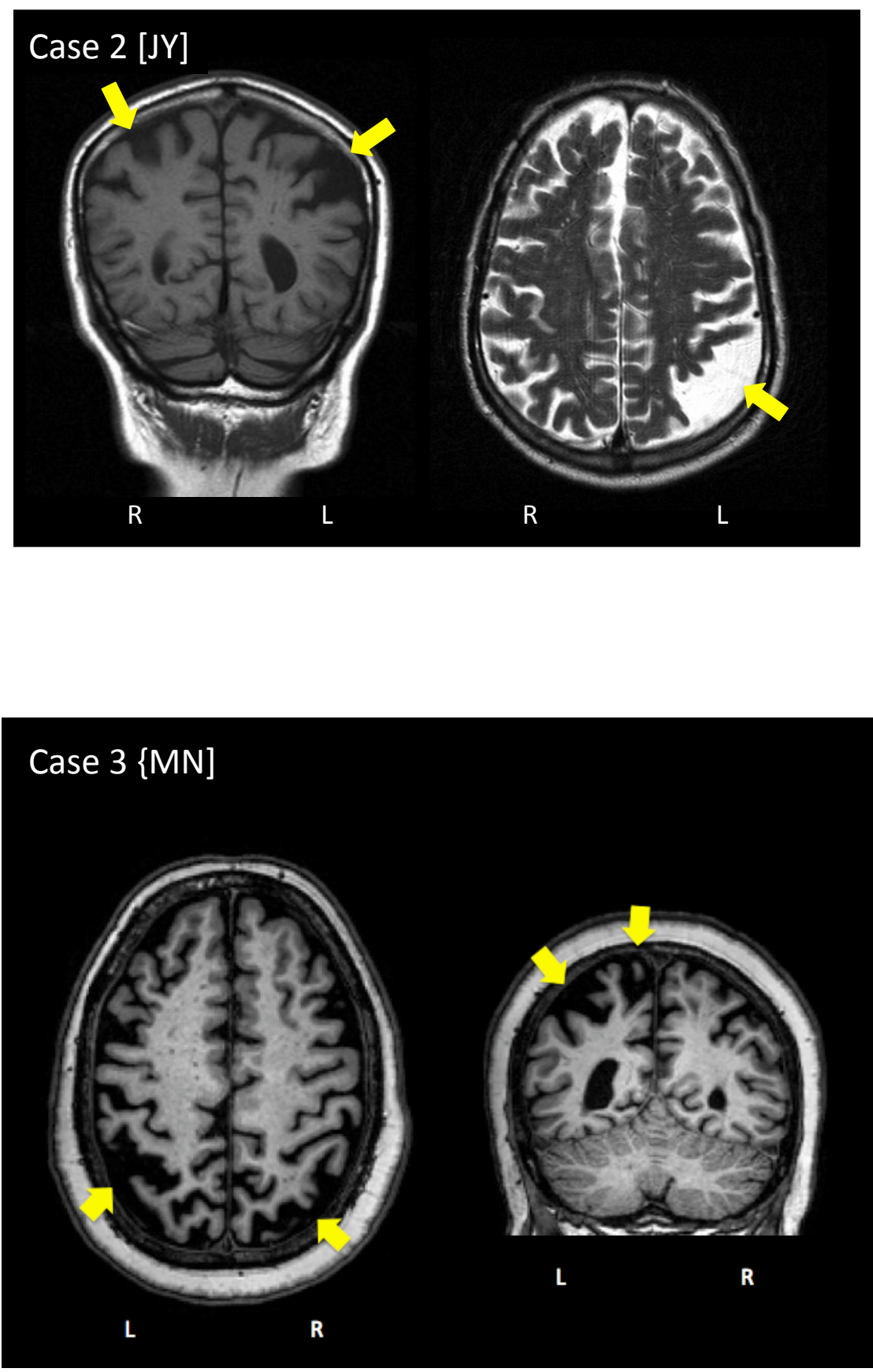
Figure 4

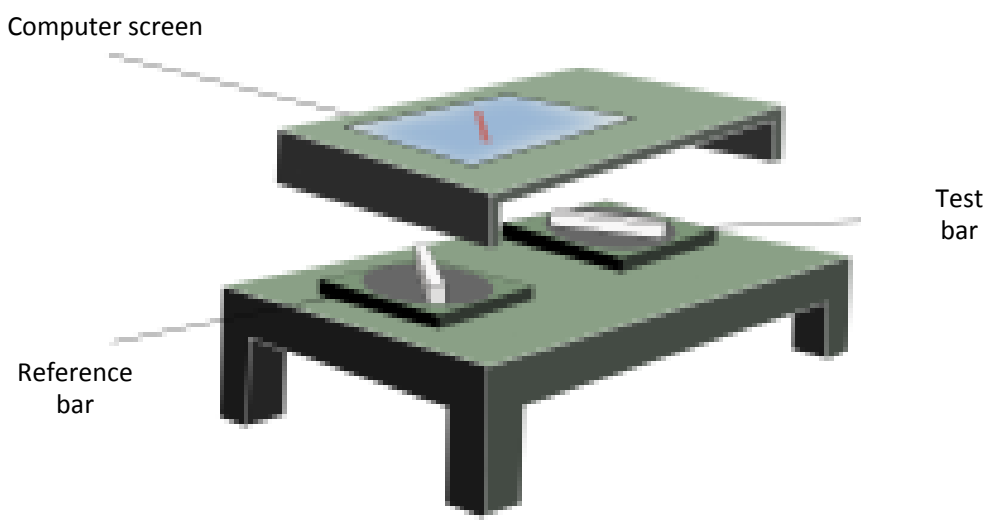

26 
Figure 5
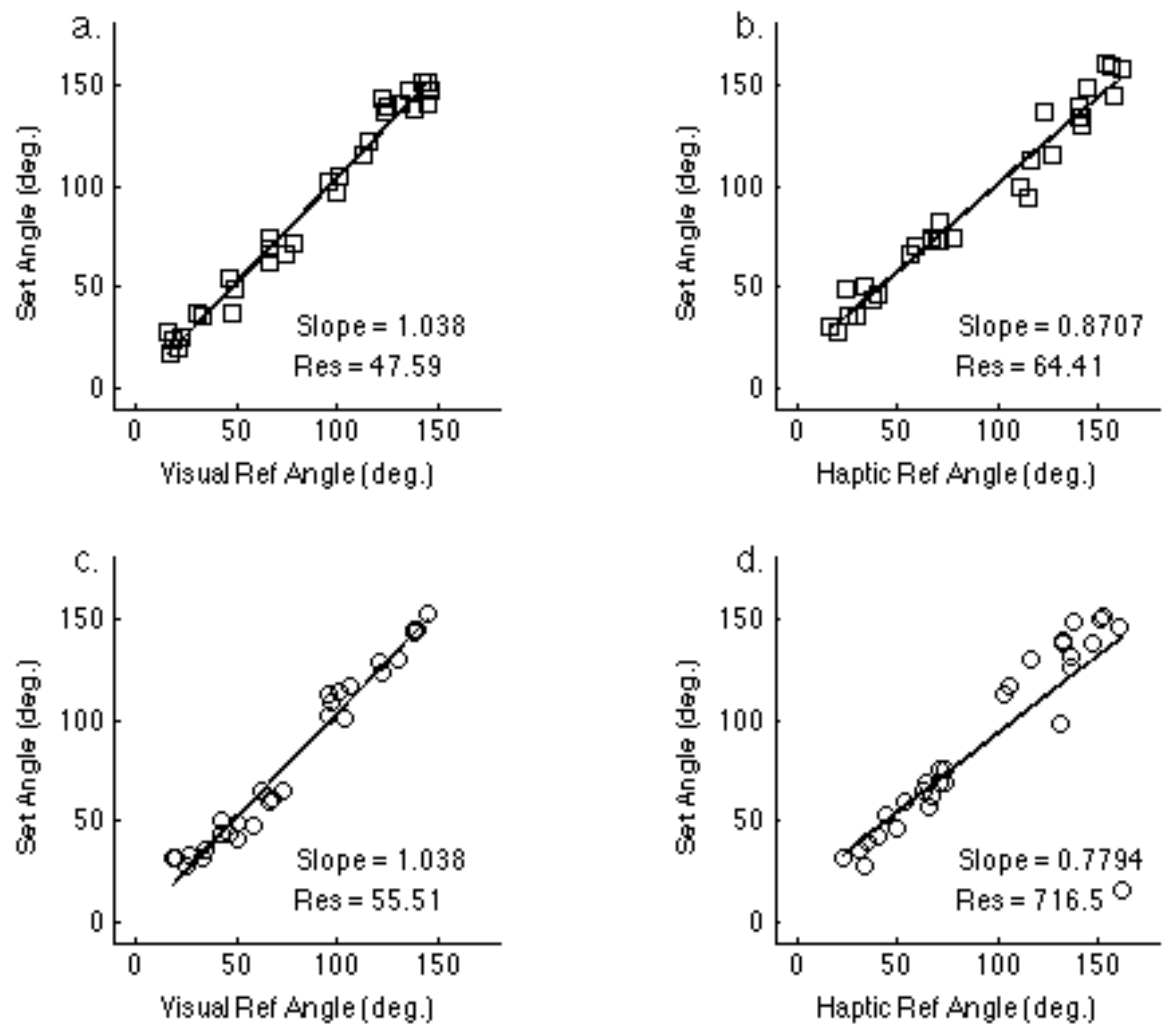
Figure 6

Case 1 (GO)
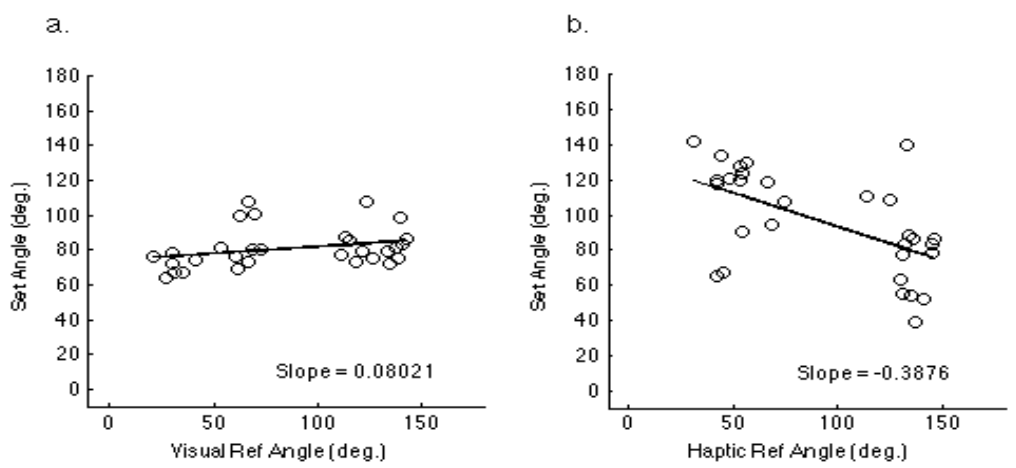

Case 2 (JY)
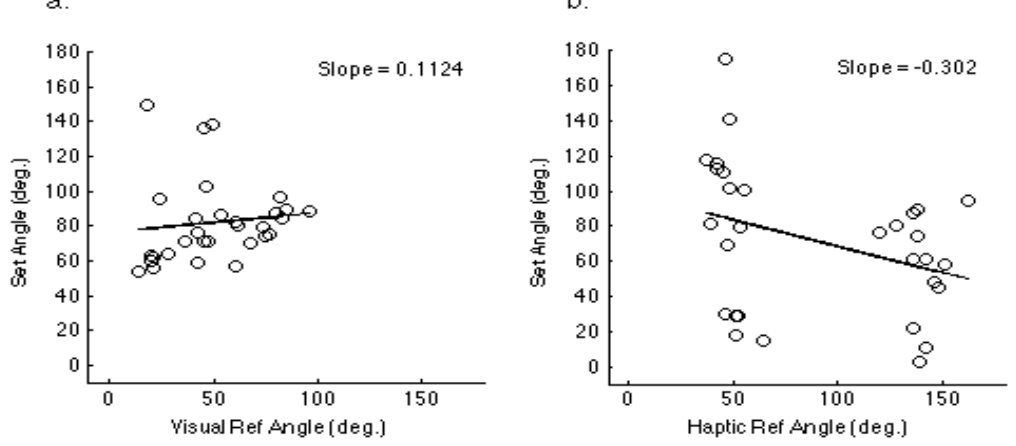

\section{Case 4 (RP)}
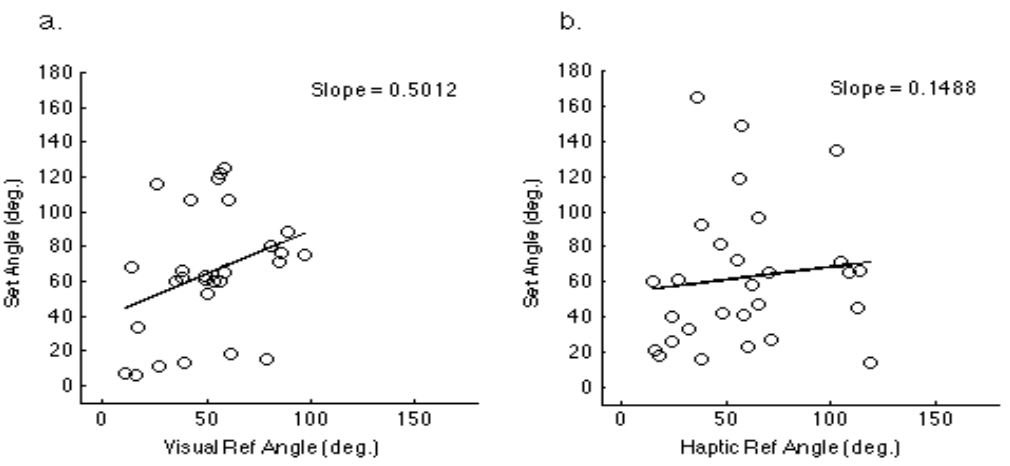
Table 1; Illustrates summary statistics for each subject that describe the degree of association between the reference bar angle (set by the experimenter on each trial) and the angle of the test bar set by the subject on each trial in the parallel match condition. Slope: refers to the linear regression slope; $\underline{\mathrm{R}}^{2}$ refers to the variance explained by predictor variable; $\mathrm{r}$ : is the Pearson correlation coefficient; r-to-Z: is Fisher's $r$ to Z transformation of the correlation coefficients; 95\% C.I.: are the lower and upper 95\% confidence intervals for the correlation coefficients; $\mathrm{t}$-val: is the Student's t-value for the correlation coefficients; and $\mathrm{p}$ val: is the $p$ value for the correlation coefficients.

\begin{tabular}{|c|c|c|c|c|c|c|c|}
\hline Subject & Task & Slope & $\mathbf{R}^{\mathbf{2}}$ & $\mathbf{r}$ & $\mathbf{r - t o - Z}$ & t-val & p-val \\
\hline 1 & $\mathrm{P}$ & 0.78 & 0.95 & 0.98 & 2.30 & 33.66 & 0.001 \\
\hline & $\mathrm{M}$ & 0.85 & 0.94 & 0.97 & 2.09 & 31.52 & 0.001 \\
\hline 2 & $\mathrm{P}$ & 0.95 & 0.93 & 0.97 & 2.09 & 27.41 & 0.001 \\
\hline & $\mathrm{M}$ & 0.95 & 0.94 & 0.97 & 2.09 & 30.41 & 0.001 \\
\hline 3 & $\mathrm{P}$ & 0.85 & 0.96 & 0.98 & 2.30 & 40.53 & 0.001 \\
\hline & $\mathrm{M}$ & 0.83 & 0.97 & 0.99 & 2.65 & 47.11 & 0.001 \\
\hline 4 & $\mathrm{P}$ & 0.90 & 0.96 & 0.98 & 2.30 & 40.27 & 0.001 \\
\hline & $\mathrm{M}$ & 0.88 & 0.97 & 0.99 & 2.65 & 47.63 & 0.001 \\
\hline 5 & $\mathrm{P}$ & 0.80 & 0.97 & 0.98 & 2.30 & 51.08 & 0.001 \\
\hline & $\mathrm{M}$ & 0.84 & 0.97 & 0.99 & 2.65 & 54.87 & 0.001 \\
\hline 6 & $\mathrm{P}$ & 0.69 & 0.97 & 0.98 & 2.30 & 41.74 & 0.001 \\
\hline & $\mathrm{M}$ & 0.71 & 0.96 & 0.98 & 2.30 & 37.58 & 0.001 \\
\hline 7 & $\mathrm{P}$ & 0.77 & 0.95 & 0.98 & 2.30 & 33.82 & 0.001 \\
\hline & $\mathrm{M}$ & 0.84 & 0.93 & 0.97 & 2.09 & 29.74 & 0.001 \\
\hline 8 & $\mathrm{P}$ & 0.77 & 0.96 & 0.98 & 2.30 & 36.64 & 0.001 \\
\hline & $\mathrm{M}$ & 0.73 & 0.97 & 0.98 & 2.30 & 43.03 & 0.001 \\
\hline Means & $\mathbf{P}$ & $\mathbf{0 . 8 1}$ & $\mathbf{0 . 9 6}$ & $\mathbf{0 . 9 8}{ }^{\dagger}$ & $\mathbf{2 . 2 7}$ & & \\
\hline & $\mathbf{M}$ & $\mathbf{0 . 8 3}$ & $\mathbf{0 . 9 6}$ & $\mathbf{0 . 9 8}$ & $\mathbf{2 . 3 5}$ & & \\
\hline \hline Patient JJ & $\mathrm{P}$ & 0.03 & $<0.1$ & 0.04 & 0.04 & 0.3 & $\mathrm{NS}$ \\
\hline & $\mathrm{M}$ & -0.07 & $<0.1$ & -0.05 & -0.05 & -0.4 & $\mathrm{NS}$ \\
\hline
\end{tabular}


Table 2; Illustrates summary statistics for each subject that describe the degree of association between the reference bar angle (set by the experimenter on each trial) and the angle of the test bar set by the subject on each trial in the parallel match condition. The upper panel contains data for the condition in which the reference bar was presented visually and the lower panel contains data for the condition in which the reference bar was presented proprioceptively. Slope: refers to the linear regression slope; VarExp\%: refers to the percentage of variance explained by predictor variable; $r$ : is the Pearson correlation coefficient; r-to-Z: is Fisher's $r$ to $\mathrm{Z}$ transformation of the correlation coefficients; $95 \%$ C.I.: are the lower and upper 95\% confidence intervals for the correlation coefficients; and p-val: is the $p$ value for the correlation coefficients.

\begin{tabular}{|c|c|c|c|c|c|c|c|}
\hline \multicolumn{7}{|c|}{ Condition: visual reference; parallel match } \\
\hline Subject & Slope & VarExp\% & r & r-to-Z & \multicolumn{2}{|c|}{ 95\% C.I. } & p-val \\
\hline 1 & 1.01 & 95.5 & 0.98 & 2.39 & 0.92 & 0.99 & 0.0001 \\
\hline 2 & 1.17 & 98.4 & 0.99 & 2.74 & 0.98 & 1.00 & 0.0001 \\
\hline 3 & 0.73 & 96.3 & 0.98 & 2.70 & 0.93 & 0.99 & 0.0001 \\
\hline 4 & 0.86 & 96.9 & 0.98 & 2.45 & 0.95 & 0.99 & 0.0001 \\
\hline 5 & 0.83 & 98.1 & 0.99 & 1.88 & 0.94 & 1.00 & 0.0001 \\
\hline 6 & 0.76 & 96.7 & 0.98 & 1.94 & 0.92 & 0.99 & 0.0001 \\
\hline 7 & 1.02 & 98.5 & 0.99 & 2.12 & 0.99 & 1.00 & 0.0001 \\
\hline 8 & 0.97 & 93.5 & 0.97 & 1.96 & 0.92 & 0.99 & 0.0001 \\
\hline 9 & 0.85 & 92.2 & 0.96 & 2.16 & 0.96 & 0.98 & 0.0001 \\
\hline 10 & 0.91 & 94.4 & 0.97 & 2.21 & 0.95 & 0.99 & 0.0001 \\
\hline 11 & 1.04 & 95.2 & 0.98 & 2.49 & 0.98 & 0.99 & 0.0001 \\
\hline 12 & 0.89 & 97.2 & 0.99 & 2.29 & 0.93 & 0.99 & 0.0001 \\
\hline Means & $\mathbf{0 . 9 2}$ & $\mathbf{9 5 . 1 3}$ & $\mathbf{0 . 9 8}$ & $\mathbf{2 . 2 7}$ & & & \\
\hline
\end{tabular}

\begin{tabular}{|c|c|c|c|c|c|c|c|}
\hline \multicolumn{7}{|c|}{ Condition: proprioceptive reference; parallel match } \\
\hline Subject & Slope & VarExp\% & r & r-to-Z & \multicolumn{2}{|c|}{ 95\% C.I. } & p-val \\
\hline 1 & 1.00 & 97.4 & 0.99 & 2.52 & 0.97 & 0.99 & 0.0001 \\
\hline 2 & 1.07 & 98.2 & 0.99 & 2.68 & 0.98 & 1.00 & 0.0001 \\
\hline 3 & 0.96 & 98.3 & 0.99 & 2.72 & 0.98 & 1.00 & 0.0001 \\
\hline 4 & 0.94 & 96.1 & 0.98 & 2.30 & 0.96 & 0.99 & 0.0001 \\
\hline 5 & 0.87 & 97.1 & 0.99 & 2.45 & 0.97 & 0.99 & 0.0001 \\
\hline 6 & 0.84 & 98.6 & 0.99 & 2.81 & 0.99 & 1.00 & 0.0001 \\
\hline 7 & 0.79 & 98.6 & 0.99 & 2.81 & 0.98 & 1.00 & 0.0001 \\
\hline 8 & 0.46 & 97.6 & 0.99 & 2.54 & 0.97 & 1.00 & 0.0001 \\
\hline 9 & 0.84 & 96.9 & 0.98 & 2.42 & 0.97 & 0.99 & 0.0001 \\
\hline 10 & 0.94 & 96.3 & 0.98 & 2.33 & 0.96 & 0.99 & 0.0001 \\
\hline 11 & 0.87 & 97.9 & 0.99 & 2.61 & 0.98 & 1.00 & 0.0001 \\
\hline 12 & 0.77 & 98.6 & 0.99 & 2.81 & 0.98 & 1.00 & 0.0001 \\
\hline Means & $\mathbf{0 . 8 6}$ & $\mathbf{9 7 . 6 1}$ & $\mathbf{0 . 9 9}$ & $\mathbf{2 . 5 8}$ & & & \\
\hline
\end{tabular}

†Note that mean Pearson correlation coefficients were calculated by first obtaining the mean Fisher's r-to-Z transformation values and then applying the inverse Fisher's r-to-Z transformation to obtain the corresponding $r$ value. 
Table 3; Illustrates summary statistics for each subject that describe the degree of associatiol between the reference bar angle (set by the experimenter on each trial) and the angle of the test bar set by the subject on each trial in the mirror symmetrical match condition. The uppe] panel contains data for the condition in which the reference bar was presented visually and the lower panel contains data for the condition in which the reference bar was presented proprioceptively. Slope: refers to the linear regression slope; VarExp\%: refers to the percentage of variance explained by predictor variable; $r$ : is the Pearson correlation coefficient; r-to-Z: is Fisher's $r$ to Z transformation of the correlation coefficients; 95\% C.I.: ar the lower and upper 95\% confidence intervals for the correlation coefficients; and p-val: is tr $\mathrm{p}$ value for the correlation coefficients.

\begin{tabular}{|c|c|c|c|c|c|c|c|}
\hline \multicolumn{7}{|c|}{ Condition: visual reference; mirror match } \\
\hline Subject & Slope & VarExp\% & r & r-to-Z & \multicolumn{2}{c|}{ 95\% C.I. } & p-val \\
\hline 1 & 1.01 & 96.7 & 0.98 & 2.39 & 0.97 & 0.99 & 0.0001 \\
\hline 2 & 1.24 & 98.3 & 0.99 & 2.74 & 0.98 & 1.00 & 0.0001 \\
\hline 3 & 0.83 & 98.2 & 0.99 & 2.70 & 0.98 & 1.00 & 0.0001 \\
\hline 4 & 0.95 & 97.1 & 0.99 & 2.45 & 0.97 & 0.99 & 0.0001 \\
\hline 5 & 0.86 & 91.1 & 0.96 & 1.88 & 0.91 & 0.98 & 0.0001 \\
\hline 6 & 0.87 & 92.1 & 0.96 & 1.94 & 0.92 & 0.98 & 0.0001 \\
\hline 7 & 0.97 & 94.4 & 0.97 & 2.12 & 0.94 & 0.99 & 0.0001 \\
\hline 8 & 0.83 & 92.3 & 0.96 & 1.96 & 0.92 & 0.98 & 0.0001 \\
\hline 9 & 0.94 & 94.9 & 0.97 & 2.16 & 0.95 & 0.99 & 0.0001 \\
\hline 10 & 0.91 & 95.3 & 0.98 & 2.21 & 0.95 & 0.99 & 0.0001 \\
\hline 11 & 1.04 & 97.3 & 0.99 & 2.49 & 0.97 & 0.99 & 0.0001 \\
\hline 12 & 1.00 & 96.0 & 0.98 & 2.29 & 0.96 & 0.99 & 0.0001 \\
\hline Means & $\mathbf{0 . 9 6}$ & $\mathbf{9 5 . 3 1}$ & $\mathbf{0 . 9 8}$ & $\mathbf{2 . 2 8}$ & & & \\
\hline
\end{tabular}

\begin{tabular}{|c|c|c|c|c|c|c|c|}
\hline \multicolumn{7}{|c|}{ Condition: proprioceptive reference; mirror match } \\
\hline Subject & Slope & VarExp\% & r & r-to-Z & \multicolumn{2}{|c|}{ 95\% C.I. } & p-val \\
\hline 1 & 0.86 & 95.5 & 0.98 & 2.52 & 0.95 & 0.99 & 0.0001 \\
\hline 2 & 1.12 & 98.4 & 0.99 & 2.68 & 0.98 & 1.00 & 0.0001 \\
\hline 3 & 0.78 & 96.3 & 0.98 & 2.72 & 0.96 & 0.99 & 0.0001 \\
\hline 4 & 0.81 & 96.8 & 0.98 & 2.30 & 0.97 & 0.99 & 0.0001 \\
\hline 5 & 0.81 & 98.1 & 0.99 & 2.45 & 0.98 & 1.00 & 0.0001 \\
\hline 6 & 0.77 & 96.7 & 0.98 & 2.81 & 0.96 & 0.99 & 0.0001 \\
\hline 7 & 0.93 & 98.5 & 0.99 & 2.81 & 0.98 & 1.00 & 0.0001 \\
\hline 8 & 0.58 & 93.5 & 0.97 & 2.54 & 0.93 & 0.99 & 0.0001 \\
\hline 9 & 0.85 & 92.2 & 0.96 & 2.42 & 0.92 & 0.98 & 0.0001 \\
\hline 10 & 0.68 & 94.4 & 0.97 & 2.33 & 0.94 & 0.99 & 0.0001 \\
\hline 11 & 0.78 & 95.2 & 0.98 & 2.61 & 0.95 & 0.99 & 0.0001 \\
\hline 12 & 1.03 & 97.2 & 0.99 & 2.81 & 0.97 & 0.99 & 0.0001 \\
\hline Means & $\mathbf{0 . 8 3}$ & $\mathbf{9 6 . 0 7}$ & $\mathbf{0 . 9 8} \dagger$ & $\mathbf{2 . 3 6}$ & & & \\
\hline
\end{tabular}

${ }^{\dagger}$ Note that mean Pearson correlation coefficients were calculated by first obtaining the mean Fisher's r-to-Z transformation values and then applying the inverse Fisher's r-to-Z transformation to obtain tr. corresponding $r$ value. 
